\title{
Mathematical modeling of the kinematics of chemical droplets when pulverizing with a sprayer based on a new two-fluid turbulent model
}

\author{
Zafar Malikov ${ }^{1 *}$, Rafik Matchanov², Azamat Yuldashev ${ }^{2}$, and Nargiza Djuraeva ${ }^{1}$ \\ ${ }^{1}$ Institute of Mechanics and Seismic Stability of Structures, Tashkent, Uzbekistan \\ ${ }^{2}$ JV LLC "AGRIKHIM", Tashkent, Uzbekistan
}

\begin{abstract}
A mathematical modeling and numerical solution of the motion of the droplets of chemical reagents outflowing from the pneumatic sprayer are developed in the article. A new efficient two-fluid turbulence model is used to simulate turbulent flow. The mass fraction of liquid in the gas stream is considered small. Therefore, the effect of the liquid phase on the dynamics of air is ignored. The "parabolize" system of hydrodynamic equations is solved for the gas flow. For this, a marching method of integrating equations is used. The Lagrange approach is used to find the droplet trajectories. It is shown that the new turbulence model can be successfully applied to study two-phase submerged jets. The solutions obtained made it possible to determine a pattern of the field treatment by chemical reagents and define the sprayer's optimal parameters.
\end{abstract}

\section{Introduction}

In the cultivation of agricultural crops, special attention is paid to the technological process of spraying plants to protect them from pests, diseases, and weeds. Hundreds of thousands of tons of pesticide working solutions turn into droplets every year. The size of the droplets determines different properties of the chemicals: the rate of sedimentation and evaporation, the degree of inertial sedimentation, wind entrainment and scattering in the surface layer of the atmosphere, wetting of various surfaces of plant cover tissues, and retention of the working fluid on them, the rate of penetration of pesticides into leaf tissue, and other properties. A narrow range of droplet sizes, optimal for each specific case, characterizes a promising technology implemented by the method of spraying, which meets the everincreasing requirements of resource-saving, high productivity, and environmental friendliness [1].

\footnotetext{
*Corresponding author: malikov.z62@mail.ru
} 
However, traditional technologies for spraying vegetative plants, widely implemented using boom-type tractor sprayers (trailed, mounted, self-propelled ones) with hydraulic flatflare (standard or anti-wearable) sprayers, do not sufficiently meet modern requirements. Consumption rates of working solutions during their operation are $100-300 \mathrm{dm}^{3} / \mathrm{ha}$, and the average droplet size is $\delta=250-500 \mu \mathrm{m}$. The consumption of pesticides, calculated for technological losses and constructive imperfection of spraying equipment, is greatly overestimated. Small droplets (less than $80 \mu \mathrm{m}$ ) are carried away outside the cultivated field, and large droplets (more than $300 \mu \mathrm{m}$ ) roll off the plants, contaminating the soil [1]. Large droplets formed in the initial area of the spray cone of fluid, as a result of coagulation, are among the main reasons for the waste of pesticides.

The increased concentration of droplets in the cones of sprayed liquid of boom sprayers causes coagulation and a substantial decrease in their number, which in turn requires tens of times more flow rate of the working fluid per unit of the treated area. In addition, with finedrop spraying of a liquid, the droplets are carried into the environment. To solve this problem, the recommended height of the nozzles on the sprayer boom $(0.5 \mathrm{~m})$ limits its travel velocity to $12 \mathrm{~km} / \mathrm{h}$, especially with a wide-grip boom. At its vibrations up to $12^{0}$, there is a danger of the edges touching the ground. Turbulent air vortices appearing behind the boom during the sprayer motion increase the sprayed liquid droplets entrainment into the environment [2].

In this regard, the study of drop dynamics is an urgent task when designing new devices for spraying chemicals. A great number of publications are devoted to the study of the dynamics and heat- and mass-transfer of droplets. For example, the dynamics of liquid-drop refrigerant during aircraft fire suppression is studied in [3]. In that article, the motion of droplets in the air at rest is considered. In contrast to that article, the authors of the current article investigate the dynamics of droplets of chemical reagents in a turbulent flow formed by a pneumatic sprayer.

The schematic diagram of this device is shown in Figure 1. The principle of operation of a sprayer is that the resulting droplets from centrifugal nozzles are carried away by an air stream and directed to the plants. As a result of the influence of the air flow on the plants, the degree of penetration of droplets onto the undersides of the leaves increases substantially. The process takes place at high Reynolds numbers [23-26]. Therefore, the air flow has a purely turbulent character. Therefore, the kinematics of droplets should be considered based on the theory of flow turbulence.

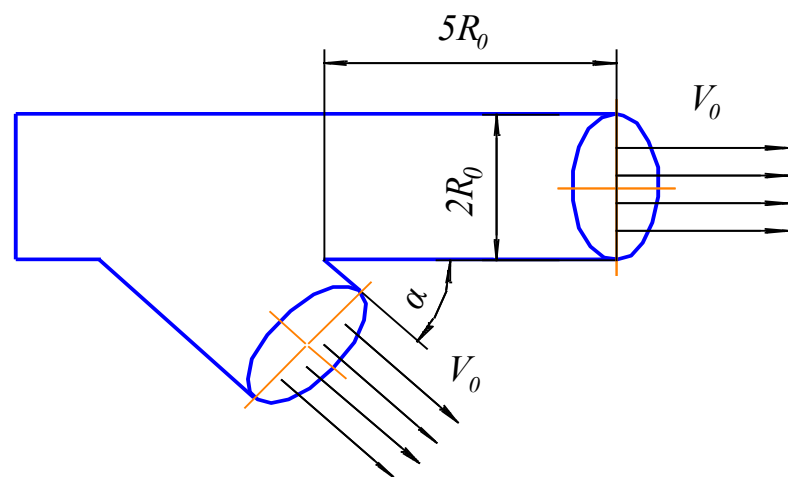

Fig. 1. Schematic diagram of the sprayer

When modeling the kinematics of droplets, it is assumed that the mass of the liquid phase per unit volume of air is much less than the mass of the air flow. This assumption greatly simplifies the task under consideration. Because in this case, the influence of the 
liquid phase on the dynamics of the air flow can be ignored, i.e. the liquid phase can be considered a passive impurity. Consequently, the problem is divided into two independent tasks: the first problem is modeling and solving the dynamics of an air turbulent jet; the second problem is based on the obtained pattern of the air velocity field at modeling and numerical study of the droplet motion.

For almost 100 years modeling a turbulent submerged jet has attracted the interest of many researchers. This is because jet streams are widely used in engineering and technological processes. It is known from the classical literature that the first major step in modeling jet streams belongs to the Prandtl theory of the mixing paths of a turbulent flow. This theory made it possible to close the system of Reynolds-averaged Navier-Stokes equations (RANS) and obtain a solution on a self-similar section, i.e. at long distances from the nozzle. However, in many situations, serious and interesting processes occur near the nozzle exit. In such cases, the use of Prandtl theory can be considered a rough estimate of the process. Currently, many different RANS turbulence models were developed for calculating turbulent jets; they allow one to obtain an adequate solution at the initial, transitional, and self-similar sections. These models include various modifications of $k-\varepsilon, k-\omega, \mathrm{SA}, \mathrm{SST}$ [4-7] and other models. Despite the many RANS models developed, virtually all of them suffer from the so-called "round jet anomaly". According to RANS models, the essence of this phenomenon is that in the self-similar zone, a solution is obtained in which the jet expands 1.5 times more than is observed in experiments. Therefore, these models require different corrections for axisymmetric jets. This indicates that the universal RANS model has not yet been created. However, a recently published article [8] presents a new approach to solving the problem of turbulence. In that article, based on the two-fluid approach, a mathematical model was obtained, which was tested for turbulence problems. The new mathematical model was tested for several turbulence problems, and the results were compared with experimental data presented in the NASA database [9]. The advantage of the new model is its ease of application for practical tasks, high accuracy and stability, and the ability to describe anisotropic turbulence. In [10], the new model was used to simulate a two-phase medium in a centrifugal air separator. The current paper shows that the new two-fluid model more adequately describes turbulent swirling two-phase flows than the complex RANS models. The developed new two-fluid model has also been successfully applied for heat transfer [11]. The results obtained in this work showed that the new two-fluid model can describe in detail the heat transfer in areas where conventional RANS models cannot do this - for example, the transition zone of a viscous layer to a turbulent one. However, the main advantage of the developed two-fluid model is that it is able to adequately describe complex anisotropic turbulences. Therefore, in this work, a study of two-phase flow is carried out based on a new two-fluid turbulence model.

\section{Materials and Methods}

Turbulent jets are widely used in engineering and various technological processes. Therefore, a large number of scientific works are devoted to the study of turbulent jets [12-19]. Turbulent jets are a classic example of turbulence, and therefore, they are one of the important tasks for the verification of turbulence models.

The study in [8] shows the possibility of representing a turbulent flow as a heterogeneous mixture of two fluids in relative motion. A summary of the essence of the new two-fluid model is also presented in the article published recently $[10,11]$. In these articles, it was shown that the system of equations of the new approach for an incompressible fluid in tensor form has the following form: 


$$
\left\{\begin{array}{l}
\frac{\partial V_{i}}{\partial x_{i}}=0 . \\
\frac{\partial V_{i}}{\partial t}+V_{j} \frac{\partial V_{i}}{\partial x_{j}}+\frac{1}{\rho} \frac{\partial p}{\partial x_{i}}=\frac{\partial}{\partial x_{j}}\left[v\left(\frac{\partial V_{i}}{\partial x_{j}}+\frac{\partial V_{j}}{\partial x_{i}}\right)-\vartheta_{j} \vartheta_{i}\right] \\
\frac{\partial \vartheta_{i}}{\partial t}+V_{j} \frac{\partial \vartheta_{i}}{\partial x_{j}}=-v_{j} \frac{\partial \bar{V}_{i}}{\partial x_{j}}+\frac{\partial}{\partial x_{j}}\left[v_{i j}\left(\frac{\partial \vartheta_{i}}{\partial x_{j}}+\frac{\partial \vartheta_{j}}{\partial x_{i}}\right)\right]+\frac{F_{f i}}{\rho}+\frac{F_{\perp i}}{\rho} \\
v_{i j}=3 v+2\left|\frac{\vartheta_{i} \vartheta_{j}}{\operatorname{def}(\vec{V})}\right| \text { for } i \neq j, v_{i i}=3 v+\frac{2}{\operatorname{div} \rho v}\left|\frac{\vartheta_{k} \vartheta_{k}}{\operatorname{def}(\vec{V})}\right| \frac{\partial \rho \vartheta_{k}}{\partial x_{k}} \\
\vec{F} f=-\rho K{ }_{f}, \vec{F} \perp=\rho C_{S} \operatorname{rot} \vec{V} \times \vec{\vartheta}
\end{array}\right.
$$

Here

$$
C_{s}=0.2, K_{f}=C_{1} \lambda_{\max }+C_{2} \frac{|d \cdot \vartheta|}{d^{2}} .
$$

In the above equations $V_{i}$ is the Reynolds-averaged flow velocity in the $i$-th direction, $p$ is the averaged pressure, $\vartheta_{i}$ is the relative velocity of the first fluid, $v$ is the kinematic viscosity of the fluid, $\rho$ is the fluid density, $v_{i j}$ is the effective molar viscosity tensor, $F_{f i}$ is the friction force arising from the relative motion of the fluids, $F_{\perp i}$ is the shear force due to the shear velocity field, $d$ is the nearest distance to the solid wall, $\lambda_{\max }$ is the largest root of the characteristic equation $\operatorname{det}(A-\lambda E)=0$, where $A$ is the matrix.

$$
A=\left|\begin{array}{ccc}
-\frac{\partial V_{1}}{\partial x_{1}} & -\frac{\partial V_{1}}{\partial x_{2}}-C_{s} \zeta_{3} & -\frac{\partial V_{1}}{\partial x_{2}}+C_{s} \zeta_{2} \\
-\frac{\partial V_{2}}{\partial x_{1}}+C_{s} \zeta_{3} & -\frac{\partial V_{2}}{\partial x_{2}} & -\frac{\partial V_{2}}{\partial x_{3}}-C_{s} \zeta_{1} \\
-\frac{\partial V_{3}}{\partial x_{1}}+C_{s} \zeta_{2} & -\frac{\partial V_{3}}{\partial x_{2}}+C_{s} \zeta_{1} & -\frac{\partial V_{3}}{\partial x_{3}}
\end{array}\right|,
$$

Constant coefficients are $C_{1}=0.7825, C_{2}=0.306$.

In the problem under consideration, the pressure is considered constant. The derivatives in the diffusion terms in the axial direction could be ignored due to their smallness. Since there are no solid walls in the problem under consideration, we can assume that $C_{2}=0$. These assumptions are common and greatly facilitate the solution to the problem; the 
equations take on a parabolic form, and the problem can be solved numerically by a marching method.

Let us reduce the system of equations (1) to a dimensionless form by relating all velocities to the jet velocity at the exit from the nozzle $U_{j e t}$, and relating the distance to the radius of the nozzle $D_{j e t} / 2$; the system is written in cylindrical coordinates. For this, we introduce the notation

$$
x_{1}=z ; x_{2}=r ; V_{1}=V_{z} ; V_{2}=V_{r} ; \vartheta_{1}=\vartheta_{z} ; \vartheta_{2}=\vartheta_{r}
$$

In the new notation, the system of equations for the two-fluid model in dimensionless form has the following form:

$$
\left\{\begin{array}{l}
\frac{\partial V_{z}}{\partial z}+\frac{\partial r V_{r}}{r \partial r}=0, \\
V_{z} \frac{\partial V_{z}}{\partial z}+V_{r} \frac{\partial V_{z}}{\partial r}=\frac{1}{\operatorname{Re}}\left(\frac{\partial^{2} V_{z}}{\partial r}+\frac{\partial V_{z}}{r \partial r}\right)-\frac{\partial r \vartheta_{r} \vartheta_{z}}{r \partial r}, \\
V_{z} \frac{\partial \vartheta_{z}}{\partial z}+V_{r} \frac{\partial \vartheta_{z}}{\partial r}=-\left(1-C_{S}\right) \frac{\partial V_{z}}{\partial r} \vartheta_{r}+\frac{\partial}{r \partial r}\left(r v_{z r} \frac{\partial \vartheta_{z}}{\partial r}\right)-K_{f} \vartheta_{z}, \\
V_{z} \frac{\partial \vartheta_{r}}{\partial z}+V_{r} \frac{\partial \vartheta_{r}}{\partial r}=-C_{S} \frac{\partial V_{z}}{\partial r} \vartheta_{z}+\frac{\partial}{r \partial r}\left(2 r v_{r r} \frac{\partial \vartheta_{r}}{\partial r}\right)-2 r v_{r r} \frac{\vartheta_{r}}{r^{2}}-K_{f} \vartheta_{r}, \\
K_{f}=C_{1} \sqrt{C_{S}\left(1-C_{S}\right)}\left|\frac{\partial V_{z}}{\partial r}\right|, v_{z r}=\frac{3}{\operatorname{Re}}+2 \frac{\left|\vartheta_{r} \vartheta_{z}\right|}{\left|\partial V_{z} / \partial r\right|}, v_{r r}=\frac{3}{\operatorname{Re}}+2 \frac{\vartheta_{r}^{2}}{\left|\partial V_{z} / \partial r\right|}
\end{array}\right.
$$

Here $z$ is the dimensionless axial coordinate, $r$ is the radial coordinate. For the numerical implementation of the system (2), accessible variables are the von Mises [20] variables $-(\xi, \psi)$ :

$$
\xi=z, V_{z}=\frac{\psi \partial \psi}{\bar{r} \partial \bar{r}}, V_{r}=\frac{\psi \partial \psi}{\bar{r} \partial \bar{z}} .
$$

The system of equations in new variables has the following form 


$$
\left\{\begin{array}{l}
\frac{\partial V_{z}}{\partial \xi}=\frac{\partial}{r \psi \partial \psi}\left(\frac{r^{2} V_{z}}{\operatorname{Re}} \frac{\partial V_{z}}{\psi \partial \psi}-r \vartheta_{r} \vartheta_{z}\right), \\
\frac{\partial \vartheta_{z}}{\partial \xi}=\frac{1-C_{S}}{\psi} \frac{\partial V_{z}}{\partial \psi} \vartheta_{r} r+\frac{\partial}{\psi \partial \psi}\left(\frac{r^{2} v_{z r} V_{z}}{\psi} \frac{\partial \vartheta_{z}}{\partial \psi}\right)-\frac{K_{f} \vartheta_{z}}{V_{z}}, \\
\frac{\partial \vartheta_{r}}{\partial \xi}=-\frac{C_{S}}{\psi} \frac{\partial V_{z}}{\partial \psi} \vartheta_{z} r+\frac{\partial}{\psi \partial \psi}\left(\frac{r^{2} v_{r r} V_{z}}{\psi} \frac{\partial \vartheta_{r}}{\partial \psi}\right)-2 v_{r r} \frac{\vartheta_{r}}{V_{z} r^{2}}-\frac{K_{f} \vartheta_{r}}{V_{z}} \\
K{ }_{f}=C_{1} \sqrt{C_{S}\left(1-C_{S}\right)} \frac{r V_{z}}{\psi}\left|\frac{\partial V_{z}}{\partial \psi}\right|, \\
r d r=\frac{\psi \partial \psi}{\bar{V}_{z}}, v_{z r}=\frac{3}{\operatorname{Re}}+2\left|\frac{\psi \vartheta_{r} \vartheta_{z}}{\partial V_{z}}\right|, v_{r r}=\frac{3}{\operatorname{Re}}+2\left|\frac{\psi \vartheta_{r} \vartheta_{r}}{\partial V_{z} / \partial \psi}\right|
\end{array} .\right.
$$

To integrate system (3), an implicit scheme with a central difference in the transverse direction was used. $\Delta \xi=0.0001, \Delta \psi=0.01$ integration steps were used. The number of nodes in the radial direction was 600. System (3) was solved at $R e=200000$. Initial conditions were set at the nozzle exit and corresponded to experimental measurements $V_{z}=1, \vartheta_{z}=0.055, \vartheta_{r}=0.028 r^{1 / 2}$.

\section{Results and Discussion}

To confirm the adequacy of the new two-fluid turbulence model, Figures 2 - 4 show a comparison of the numerical results of the system (3) with the experimental results. Figure 2 shows the profiles of dimensionless velocities in various sections for the initial and transitional sections of the jet. Here the experimental data were obtained from the NASA database [9].

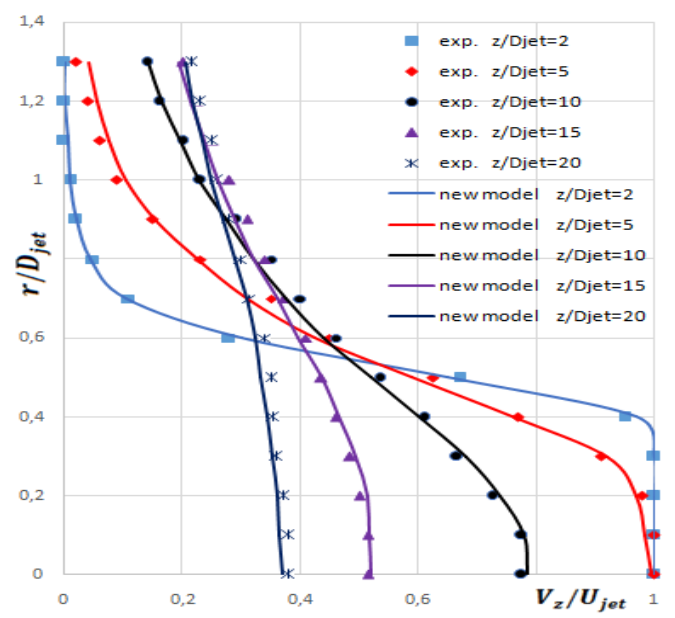

Fig. 2. Profiles of dimensionless longitudinal velocity in various sections for the initial and transitional sections of an axisymmetric jet. 
Figure 3 shows the dependence of the dimensionless axial velocity on the distance to the nozzle. Here the experimental data were also obtained from the database given in [9]. Figures 2 and 3 show the agreement of the results of the new two-fluid model with the experimental data in the initial and transitional sections.

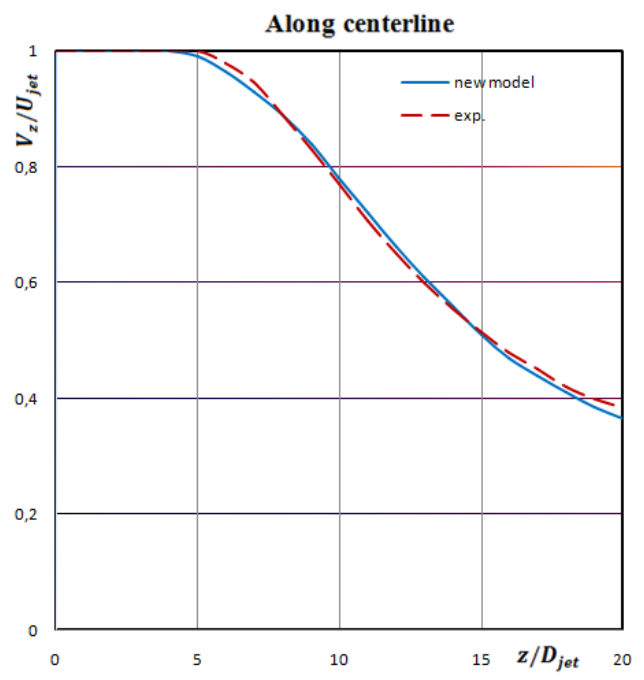

Fig.3. The dependence of the dimensionless axial longitudinal flow velocity on the distance to the nozzle.

The airflow rates obtained make it possible to determine the trajectory of reagent droplets. The set task is three-dimensional because gravity forces act on the droplets. As a result, the symmetry of the droplet motion relative to the axis is maintained. Therefore, we consider the motion of droplets coming out of the nozzles located in a vertical plane along the nozzle diameter. The resulting droplet trajectories from these nozzles give us a pattern of the sprayed area. The trajectory of droplets from the lower nozzle gives us the near boundary, and the trajectory of droplets from the upper nozzle gives us the far boundary of the treated zone. When simulating the kinematics of reagent droplets, we ignore the change in their size due to evaporation. This assumption is justified by the fact that the characteristic time for the evaporation of droplets of a size of the order of $300 \mu \mathrm{m}$ during the processing season is substantially longer than the characteristic time for the droplets to fall to the plants. The process of coagulation of droplets is also not taken into account in mathematical modeling of the kinematics of droplets. Under these assumptions, the timeaveraged equations of motion for droplets has the following form:

$$
\left\{\begin{array}{l}
\frac{d \vartheta_{d z}}{d t}=k\left(V_{\mathrm{z}}-V_{d z}\right), \\
\frac{d \vartheta_{d r}}{d t}=k\left(V_{\mathrm{r}}-V_{d r}\right)-g, \\
\frac{d z_{d}}{d t}=\vartheta_{d z}, \\
\frac{d r_{d}}{d t}=\vartheta_{d r}
\end{array}\right.
$$


In these equations $\vartheta_{d z}$ and $\vartheta_{d r}$ are the axial and radial velocities of the droplet, respectively; $z_{d}$ and $r_{d}$ are the longitudinal and radial coordinates of the droplet, respectively; $k$ is the coefficient of interaction between the droplet and the air, $g$ is the acceleration of gravity. The nozzle exit section $r=R_{0}$ corresponds to the location of the upper nozzle, and the section $r=-R_{0}$ corresponds to the lower nozzle.

Since the flow around large droplets is considered, the flow regime can be turbulent. Therefore, the Klyachko formula is used, which describes well the coefficient of interaction between phases in a wide range for the Reynolds number $R e_{d}$ [21]:

$$
k=\frac{24}{\operatorname{Re}_{d}}\left(1+0,17 \operatorname{Re}_{d}^{2 / 3}\right), \operatorname{Re}_{d}=\frac{\left|\vec{V}-\overrightarrow{V_{d}}\right| \delta}{v}
$$

In this expression, $\rho^{0}$ is the density of the fluid droplets, $\delta$ is the "effective" diameter of the droplet, and $\mu$ is the dynamic viscosity of air.

Thus, expressions (5) and the equation system (4) are mathematical models of the droplet motion. In this article, the systems of equations (4) were integrated using Euler's numerical method with second-order recalculation of accuracy [22].

Figures 4 and 5 show the trajectories of droplets exiting the sprayer by an air stream.

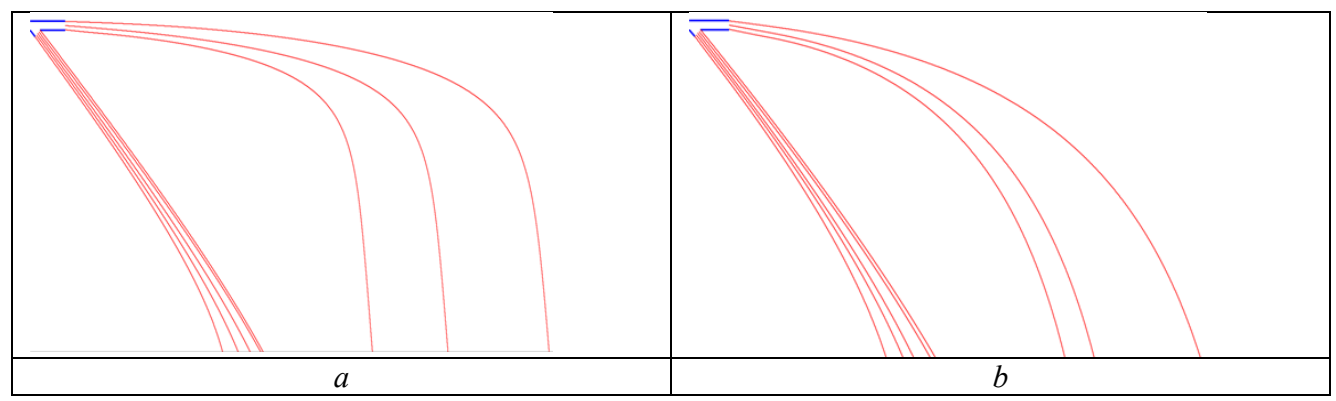

Fig. 4. Droplet trajectories with diameters: a) $300 \mu \mathrm{m}$ and b) $500 \mu \mathrm{m}$ with a second nozzle angle of $45^{0}$ to the horizon.

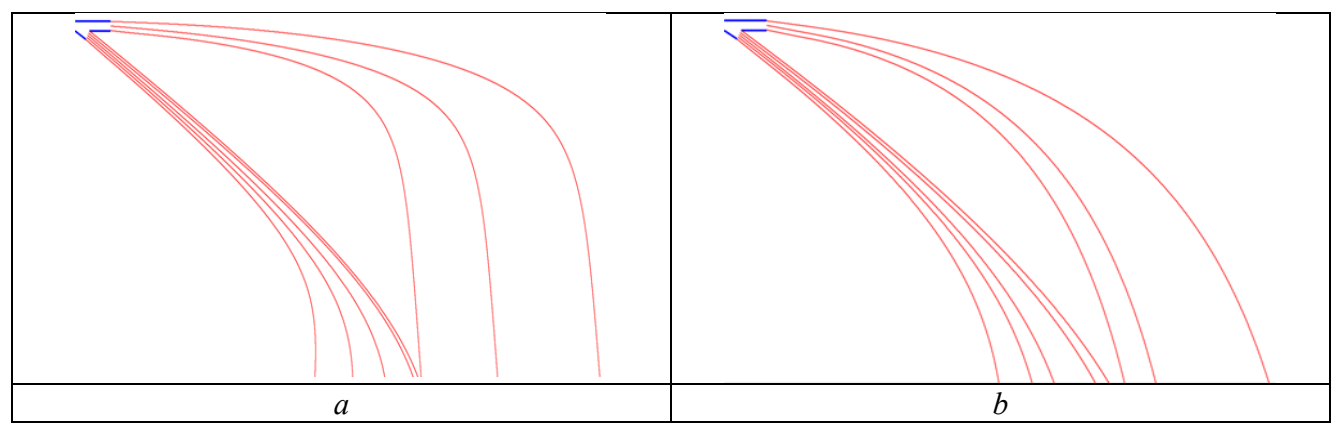

Fig. 5. Droplet trajectories with diameters: a) $300 \mu \mathrm{m}$ and b) $500 \mu \mathrm{m}$ with a second nozzle angle of $30^{0}$ to the horizon 
In these figures, length dimensions are given relative to the diameter of the nozzles. The sprayer consists of two nozzles. One nozzle is located horizontally, and the second nozzle is at an angle to the horizon. The second nozzle is located at a distance $l=5 R_{0}$ from the first nozzle. Figure 4 shows the trajectories of droplets with diameters of a) $300 \mu \mathrm{m}$ and b) 500 $\mu \mathrm{m}$ with a second nozzle angle of $45^{\circ}$ to the horizon.

As seen from these figures, the droplets cover the fall surface inhomogeneously. There is a zone where reagent droplets do not fall. Therefore, to optimize irrigation, the angle of the second (side) nozzle was adjusted. Figure 5 shows the trajectories of droplets with diameters of a) $300 \mu \mathrm{m}$ and b) $500 \mu \mathrm{m}$ with a second nozzle angle of $30^{\circ}$.

The results obtained show that when the angle of the lower nozzle is located at an angle of $30^{\circ}$ to the horizon, the uniformity of treatment by chemical reagents is substantially improved.

The calculations were conducted at an air velocity at the nozzle exit of $50 \mathrm{~m} / \mathrm{s}$. The radius of the nozzles was $R_{0}=0.1 \mathrm{~m}$.

\section{Conclusion}

1. In the article, to simulate the dynamics of a two-phase flow, a new effective two-fluid model of turbulence was used; the model made it possible to obtain solutions for a twophase jet for the initial, transitional, and self-similar flow sections.

2. Based on the mathematical model of the two-phase jet, the optimal angle of the lower nozzle of the sprayer was determined.

3. The mathematical model considered can be used to design various devices where a twophase flow occurs in a submerged jet.

\section{References}

1. Nikitin N.V., Spiridonov Yu.Ya., Shestakov V.G. Scientific and practical aspects of the technology of application of modern herbicides in crop production, Moscow: RASHN. p.189. (in Russian). (2010)

2. Nikitin N.V., Spiridonov Yu.Ya., Abubikerov V.A. et al. Anti-entrainment technology of introducing herbicides of a new generation. Plant Protection Bulletin - VIZR, (3). pp. 47-55. (in Russian). (2008).

3. Arkhipov V.A., Matvienko O.V., Zharova I.K., Maslov E.A., Perfilieva K.G., Bulavko A.M. Modeling of the dynamics of a liquid-droplet refrigerant under aerial firefighting. Tomsk State University. Journal of Mathematics and Mechanics, 62, pp. 68-78.(in Russian). (2019).

4. Launder B.E., Spalding D.B. Lectures in Mathematical Models of Turbulence, London: Academic Press, p 169. (1972).

5. Wilcox, D. C, Reassessment of the Scale-Determining Equation for Advanced Turbulence Models, AIAA Journal. 26. (11). pp. 1299-1310. DOI 10.2514/3.10041. (1988).

6. Spalart P. R., Allmaras S. R. A one-equation turbulence model for aerodynamic flows, AIAA Paper 0439. DOI 10.2514/6.1992-439.( 1992).

7. Menter F. R. Zonal two-equation k- $\omega$ turbulence models for aerodynamic flows, AIAA Paper 1993-2906. . DOI 10.2514/6.1993-2906. (1993)

8. Malikov Z. Mathematical model of turbulence based on the dynamics of two fluids, Applied Mathematical Modeling 82 (2020) pp 409-436. DOI 10.1016/j.apm.2020.01.047. (2020) 
9. Turbulence modeling Resource. NASA Langley Research Center, http://turbmodels.larc.nasa.gov.

10. Malikov Z.M., Madaliev M.E. Numerical modeling of two-phase flow in a centrifugal separator, ISSN 0015-4628, Fluid Dynamics, 2020, 55, (8), pp. 1012-1028. (C) Pleiades Publishing, Ltd., (2020).

11. Malikov Z.M. "Mathematical model of turbulent heat transfer based on the dynamics of two fluids". Applied Mathematical Modeling, 91, pp.409-436.

12. Bridges, J.,Wernet, M. P. "Establishing Consensus Turbulence Statistics for Hot Subsonic Jets," AIAA Paper 2010, p 3751, 16th AIAA/CEAS Aeroacoustics Conference, Stockholm, Sweden, June, (2010)

13. Hussein H.J., Capp S.P., George W.K. Velocity measurements in a high-Reynoldsnumber, momentum-conserving, axisymmetric, turbulent jet, J. Fluid Mech. (258), pp. 31-75, (1994).

14. Wygnanski I., Fiedler H. Some measurements in the self-preserving jet, J. Fluid. Mech., 38, pp. 577-612, (1969).

15. Darisse A., Lemay J., Benaissa A. LDV measurements of well converged third order moments in the far field of a free turbulent round jet // Exp. Thermal Fluid Sci. 44, pp. 825-833, (2013).

16. Gutmark E., Wygnanski I.J. The planar turbulent jet, J. Fluid Mech., 73, pp. 465-495, (1976).

17. Ramaprian B.R., Chandrasekhara M.S. LDA measurements in plane turbulent jets, J. Fluids Eng. 107, pp. 264-271, (1985).

18. Bradbury L.J.S. The structure of a self-preserving turbulent plane jet, J. Fluid Mech, 23, pp. 31-64, (1965).

19. Heskestad G. Hot-wire measurements in a plane turbulent jet, J. Appl. Mech., 32, pp. 721-734, (1965).

20. Von Mises R. “Bernerkungen zur Hydrodynamik”. Z. Angew. Math. u. Mech., 7, p 425 (1927).

21. Klyachko L.S. On one feature of the mechanism of the flow effect on solid bottom particles. Justification of the minimum air velocities in the air ducts of dedusting ventilation units. - In: Theory and practice of dedusting ventilation. L.: Profizdat, 1952, pp. 60-73 (Tr. LIOT: Issue 5). (in Russian). (1952).

22. Anderson D., Tannehill J., Pletcher R. Computational fluid mechanics and heat transfer, 1. Moscow. "Mir" .p 107. (1990).

23. Bazarov D., Norkulov B., Vokhidov O., Uljaev F., Ishankulov, Z. Two-dimensional flow movement in the area of protective regulatory structures. IOP Conf. Ser. Mater. Sci. Eng. 890, 012162 (2020)

24. Bazarov D. and Vokhidov O. Extinguishing Excess Flow Energy in Spillway Structures. In book: Proceedings of EECE 2020, LNCE 150, pp. 535-545, (2021) DOI: 10.1007/978-3-030-72404-7_52

25. Krutov A., Choriev R., Norkulov B., Mavlyanova D. and Shomurodov A. Mathematical modelling of bottom deformations in the kinematic wave approximation. IOP Conf. Ser. Mater. Sci. Eng. 1030, 012147 (2021).

26. Krutov A., Norkulov B., Uljaev F., and Jamalov F. Results of a numerical study of currents in the vicinity of a damless water intake. IOP Conf. Ser. Mater. Sci. Eng. 1030, 012121 (2021). 